\title{
Straightforward Glycoengineering Approach to Site-Specific Antibody-Pyrrolobenzodiazepine Conjugates
}

Pamela Thompson, ${ }^{* \dagger}{ }^{\dagger}$ Ebele Ezeadi, ${ }^{\S}$ Ian Hutchinson, ${ }^{\S}$ Ryan Fleming, ${ }^{\dagger}$ Binyam Bezabeh, ${ }^{\dagger}$ Jia Lin,${ }^{\dagger}$ Shenlan Mao, ${ }^{\ddagger}$ Cui Chen, ${ }^{\ddagger}$ Luke Masterson, ${ }^{\S}$ Haihong Zhong, ${ }^{\ddagger}$ Dorin Toader, ${ }^{\dagger}$ Philip Howard, ${ }^{\S}$ Herren $\mathrm{Wu},{ }^{\dagger}$ Changshou Gao, ${ }^{*}, \dagger$ and Nazzareno Dimasi ${ }^{*}{ }^{\dagger}$

${ }^{\dagger}$ Antibody Discovery and Protein Engineering and ${ }^{\ddagger}$ Oncology Research, MedImmune, One MedImmune Way, Gaithersburg, Maryland 20878, United States

${ }^{\S}$ QMB Innovation Centre, Spirogen, 42 New Road, E1 2AX London, U.K.

Supporting Information

ABSTRACT: Antibody-drug conjugates (ADCs) have become a powerful platform to deliver cytotoxic agents selectively to cancer cells. ADCs have traditionally been prepared by stochastic conjugation of a cytotoxic drug using an antibody's native cysteine or lysine residues. Through strategic selection of the mammalian expression host, we were able to introduce azide-functionalized glycans onto a homogeneously glycosylated anti-EphA2 monoclonal antibody in one step. Conjugation with an alkyne-bearing pyrrolobenzodiazepine dimer payload (SG3364) using copper-catalyzed click chemistry yielded a site-specific ADC with a drug-to-antibody ratio (DAR) of four. This $\mathrm{ADC}$ was compared with a glycoengineered DAR two site-specific $\mathrm{ADC}$, and both were found to be highly potent against EphA2-positive human prostate cancer cells in both an in vitro cytotoxicity assay and a murine tumor xenograft model.

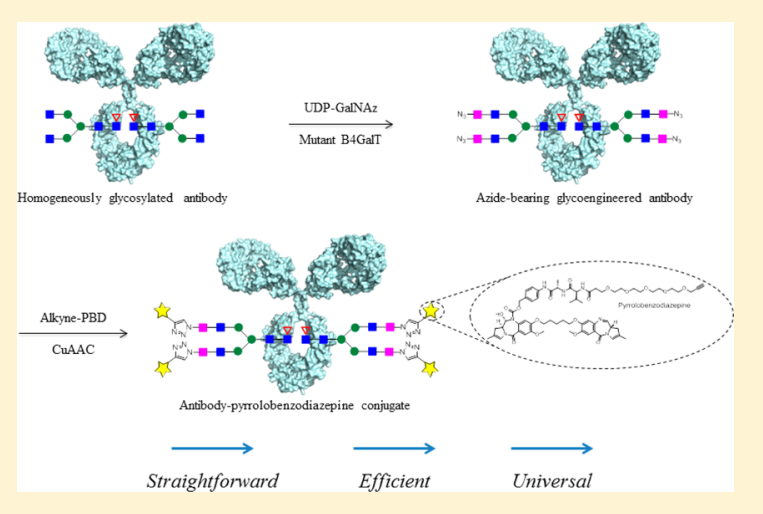

KEYWORDS: Antibody-drug conjugates, pyrrolobenzodiazepine, SG3364, click chemistry, carbohydrate remodeling, site-specific conjugation

A ntibody-drug conjugates (ADCs) are an important class of biologics, which combine the potency of cytotoxic drugs with the specificity of antibodies. To date, there are two clinically approved ADCs, Adcetris and Kadcyla, both of which are stochastically conjugated through either cysteine or lysine residues. $^{1,2}$ In addition to the knowledge gained through preclinical and clinical studies, advances in protein engineering and bioorthogonal chemistry have shifted the focus to generating site-specific ADCs, which yield homogeneous products that demonstrate improved in vivo properties. ${ }^{3-6}$

Human immunoglobulins have a conserved glycosylation site in the $\mathrm{CH} 2$ domain of the heavy chain at position N297, making it an attractive target for generating site-specific antibody-drug conjugates. ${ }^{7}$ However, since glycosylation is a heterogeneous post-translational modification, ${ }^{8-10}$ remodeling of the glycan is often required before conjugation of the payload. Many reported strategies involve chemical drug conjugation following a multienzymatic process to install either natural or synthetic carbohydrate analogues, such as CMP-9azido sialic acid and UDP-GalNAz derivatives (Figure 1), onto the antibody glycan. ${ }^{11-13}$ Pan et al. described a multistep approach in which first the antibody glycan was remodeled to contain sialic acid residues, which were then chemically oxidized with sodium periodate and conjugated with an auristatin via oxime chemistry. ${ }^{14}$ Boons et al. reported a

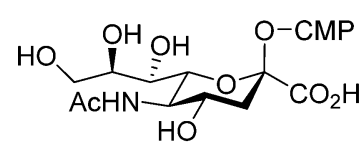

CMP-sialic acid

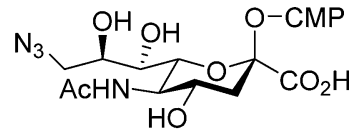

CMP-9-azido sialic acid

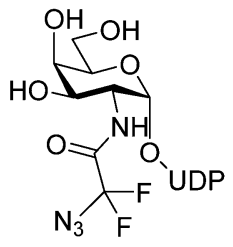

UDP-F 2 -GalNAz

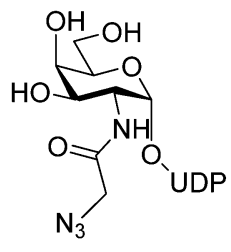

UDP-GalNAz
Figure 1. Nucleotide sugars used in chemoenzymatic ADC strategies.

Special Issue: Antibody-Drug Conjugates and Bioconjugates

Received: July 19, 2016

Accepted: September 19, 2016

Published: September 20, 2016 
multienzymatic process for the conversion of the G0F glycoform to G2F, followed by installation of C9-azido sialic acid residues (Figure 1). Subsequent conjugation of a doxorubicin payload using strain-promoted azide-alkyne cycloaddition (SPAAC) resulted in an ADC with a drug-toantibody ratio (DAR) of $4 .{ }^{15}$ Recently, SynAffix reported a strategy in which the $\mathrm{N}$-glycan was partially deglycosylated by endoglycosidase S (EndoS), and fluorinated GalNAz derivatives (Figure 1) were installed enzymatically using a mutant $\beta 1,4$ galactosyltransferase (GalT(Y289L)), ${ }^{13,16}$ followed by conjugation of monomethyl auristatin $\mathrm{F}$ to the antibody using SPAAC, yielding ADCs with a DAR of $2 .^{17}$

Herein we describe a one-step chemoenzymatic method to generate $\mathrm{ADCs}$ with a drug-to-antibody ratio of 4 using an alkynyl pyrrolobenzodiazepine (PBD) dimer payload (SG3364, Scheme 1) conjugated to an azide-modified GalNAc (GalNAz,

Scheme 1. Synthesis of SG3364 ${ }^{a}$
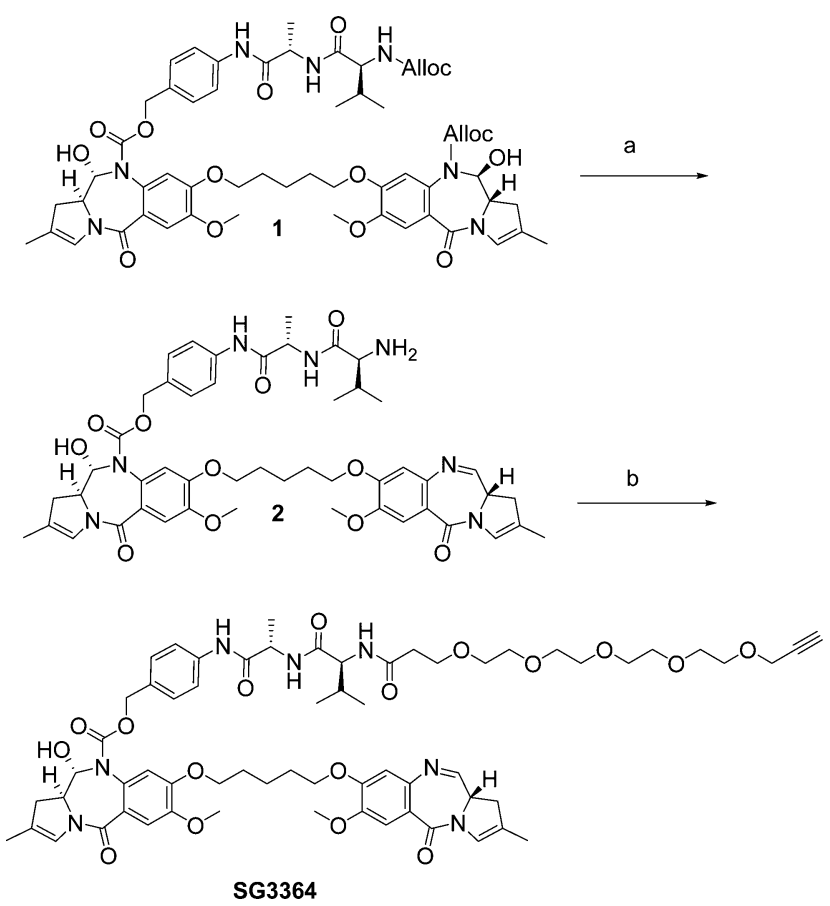

${ }^{a}$ Reagents and conditions: (a) $\mathrm{Pd}\left(\mathrm{PPh}_{3}\right)_{4}$, pyrrolidine, DCM, (b) propargyl- $\mathrm{PEG}_{5}$-acid, EDCI, $2 \% \mathrm{MeOH}: \mathrm{CHCl}_{3}, 43 \%$.

Figure 1) using copper-catalyzed click chemistry (Figure 2A,C). Using this approach and an EndoS glycan remodeling strategy (Figure 2B), ${ }^{17}$ ADCs with drug loads of four and two were respectively prepared, analytically characterized, and evaluated for their in vitro and in vivo activity.

Unlike other methods that generate DAR $4 \mathrm{ADCs},{ }^{13,15}$ our approach requires no glycan remodeling of the antibody prior to the enzymatic introduction of GalNAz, which facilitates the manufacturing process of ADCs. While most monoclonal antibodies (mAbs) are expressed with a heterogeneous glycoprofile, homogeneous G0/G0F glycosylation of an antiEphA2 antibody (1C1-G0F) was achieved by recombinant expression in a Chinese Hamster Ovary (CHO)-LEC8 host system (Supplementary Figure 2B). ${ }^{18,19}$ Precise installation of four azides per antibody (two per heavy chain) was then achieved after overnight incubation of 1C1-G0F with GalT(Y289L) and UDP-GalNAz in the presence of $\mathrm{MnCl}_{2}$ (Figure

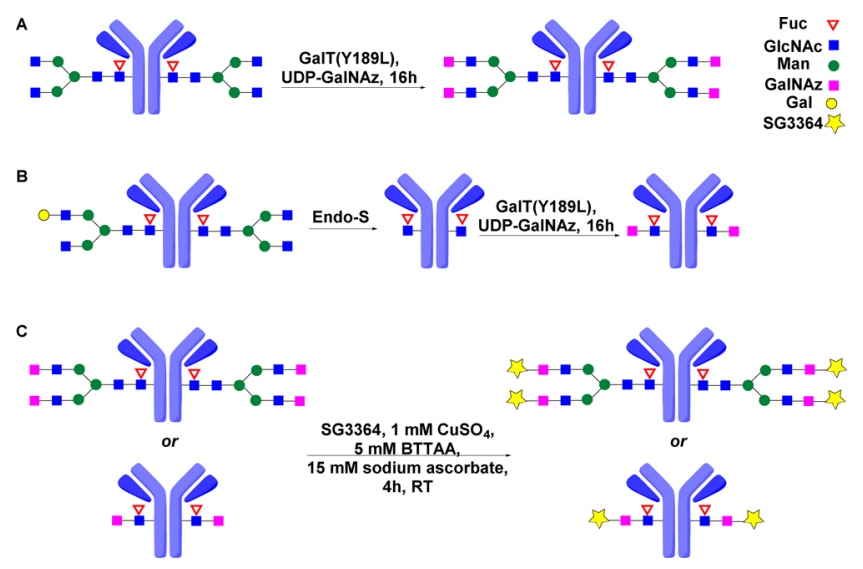

Figure 2. Glycoengineering approach to generating DAR 2 and DAR 4 ADCs. (A) Homogeneous GOF glycosylated mAb expressed in $\mathrm{CHO}$ LEC8 cells allows introduction of four azides per antibody. (B) Treatment of heterogeneously glycosylated $\mathrm{mAb}$ with Endo-S results in the installation of two azides per antibody. (C) Conjugation of SG3364 using copper-catalyzed click chemistry.

2A). Complete installation of two GalNAz units onto each heavy chain was confirmed by reduced LC/MS (Supplementary Figure 2D).

A wild-type $1 \mathrm{C} 1$ antibody with heterogeneous glycosylation was obtained using a HEK293 transient expression system, as described by Thompson et al., ${ }^{6}$ and treated with EndoS, which resulted in trimming of the $N$-glycan to unveil a free GlcNAc (Figure 2B). Two GalNAz units were transferred onto the partially deglycosylated 1C1-EndoS using GalT(Y289L), which was confirmed using mass spectrometry (Supplementary Figures 2A,C,F).

Compound 1 was synthesized as described by Tiberghien et al. $^{20}$ The N10 and N-terminal alloc groups of compound 1 were deprotected using $\operatorname{Pd}(0)$ with pyrrolidine as an allyl scavenger. The resulting amine-imine $\mathbf{2}$ was directly used in the next step without further purification. Therefore, crude 2 was coupled to propargyl-PEG5-acid with EDCI to give SG3364 in 43\% yield over two steps (Scheme 1).

With SG3364 in hand, we set out to generate ADCs with either DAR 2 or DAR 4. To this end, the GalNAz-modified antibodies were conjugated with SG3364 in the presence of $\mathrm{CuSO}_{4}, \mathrm{BTTAA}^{21}$ and sodium ascorbate for $4 \mathrm{~h}$ at room temperature (Figure 2C). Excellent conjugation efficiency was observed (Table 1), as detected by both reduced LC/MS and

Table 1. Analytical Characterization of $\mathrm{ADCs}^{a}$

\begin{tabular}{cccc}
\multicolumn{1}{c}{ ADC } & $\%$ monomer & $\% \mathrm{HC}$ conjugated & DAR \\
1C1-EndoS-GalNAz-SG3364 & $98 \%$ & $97.5 \%$ & 1.95 \\
1C1-G0F-GalNAz-SG3364 & $97 \%$ & $8 \% \mathrm{HC}+1$ & 3.84 \\
& & $92 \% \mathrm{HC}+2$ &
\end{tabular}

${ }^{a}$ Monomeric content assessed by SEC-HPLC. DAR calculated by reduced RP-LC/MS.

reduced RP-HPLC (Supplementary Figures 2 and 3). Both glycoengineered $\mathrm{ADCs}$ were highly monomeric, as determined by SEC-HPLC (Table 1), and exhibited a drug-load dependent hydrophobic character, as demonstrated by HIC-HPLC (Figure 3).

As expected, both glycoengineered ADCs were highly potent in vitro in a cell killing assay using the EphA2-positive PC3 cell 


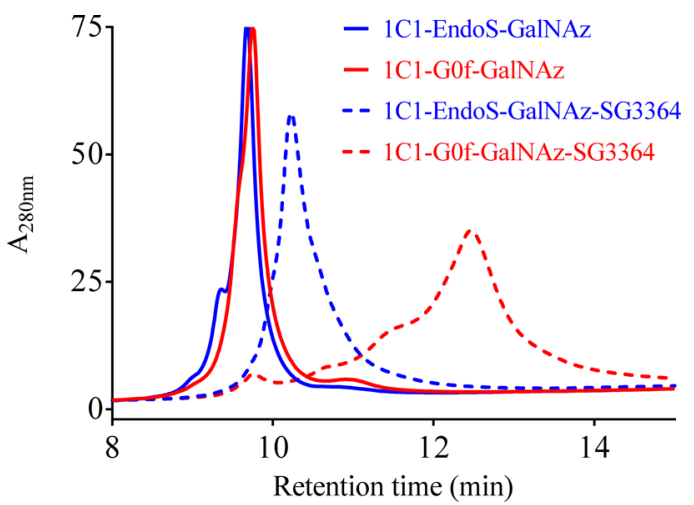

Figure 3. Hydrophobicity profile for DAR 2 and DAR 4 glycoengineered ADCs.

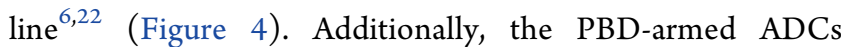
demonstrated specific and potent activity in a murine xenograft PC3 prostate cancer model ${ }^{6,22}$ (Figure 5).

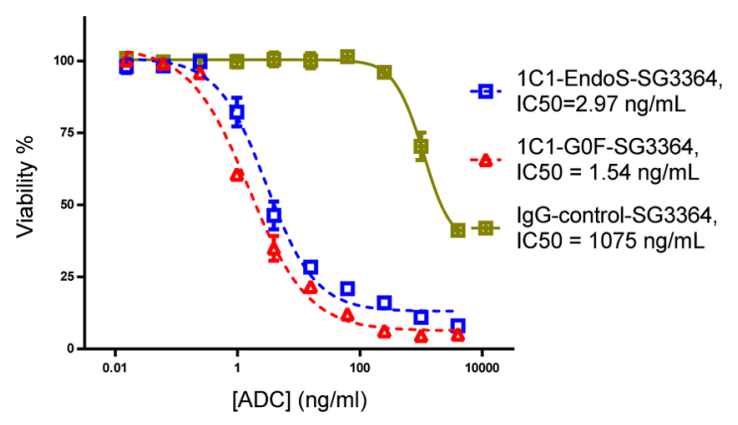

Figure 4. Pyrrolobenzodiazepine dimer ADCs are potent in vitro in EphA2-positive PC3 cells. 1C1-ADCs have potent cytotoxicity, whereas negative control IgG-control $\mathrm{ADC}$ does not.

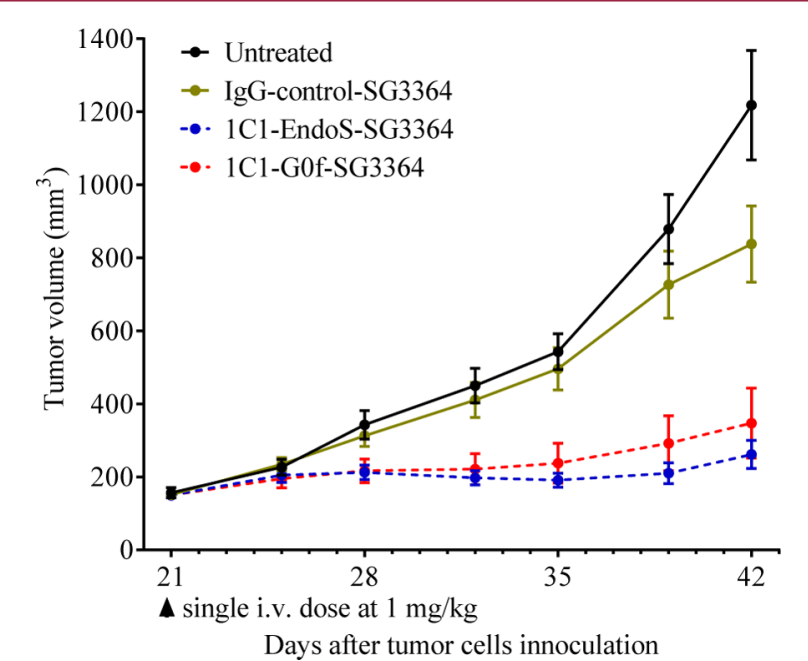

Figure 5. Pyrrolobenzodiazepine dimer glycoengineered ADCs are efficacious in an in vivo PC3 tumor xenograft model.

Next, we set out to determine the in vitro stability of the glycoengineered ADCs using a previously established method. ${ }^{6,22}$ After 3 days incubation at $37{ }^{\circ} \mathrm{C}$ in mouse serum, no payload loss was observed (Supplementary Figure 4).

In summary, we have demonstrated that it is possible to generate homogeneous ADCs using a glycoengineering strategy. By careful selection of the expression host system, homogeneous G0F glycosylation of a monoclonal antibody was achieved. This allowed for a one-step process to install GalNAz onto the mAb and generate an ADC loaded with four SG3364 PBD dimers by copper-catalyzed click chemistry. This straightforward approach is highly versatile, does not require antibody engineering, and is broadly applicable to the preparation of any site-specific ADC containing any payload class.

\section{ASSOCIATED CONTENT}

\section{Supporting Information}

The Supporting Information is available free of charge on the ACS Publications website at DOI: 10.1021/acsmedchemlett.6b00278.

Detailed experimental procedures with analytical data for enzyme, mAbs, ADCs, and payload (PDF)

\section{AUTHOR INFORMATION}

\section{Corresponding Authors}

*E-mail: thompsonpam@medimmune.com.

*E-mail: gaoc@medimmune.com.

*E-mail: dimasin@medimmune.com.

\section{Author Contributions}

The manuscript was written through contributions of all authors. All authors have given approval to the final version of the manuscript.

\section{Funding}

This study was supported by MedImmune.

\section{Notes}

The authors declare the following competing financial interest(s): All authors are employees of MedImmune and are stockholders of AstraZeneca.

\section{ABBREVIATIONS}

$\mathrm{ADC}$, antibody-drug conjugate; $\mathrm{PBD}$, pyrrolobenzodiazepine; DAR, drug-to-antibody ratio; SPAAC, strain-promoted azidealkyne cycloaddition; $\mathrm{CHO}$, Chinese hamster ovary; HEK, human embryonic kidney; LC/MS, liquid chromatographymass spectrometry; RP-HPLC, reverse-phase high performance liquid chromatography; SEC, size exclusion chromatography; $\mathrm{HIC}$, hydrophobic interaction chromatography; $\mathrm{HC}$, heavy chain; EDCI, $\mathrm{N}$-(3-(dimethylamino)- propyl)- $\mathrm{N}^{\prime}$-ethylcarbodiimide hydrochloride; DCM, dichloromethane; $\mathrm{MeOH}$, methanol; PEG, polyethylene glycol; Endo-S, endoglycosidase-S; $\mathrm{PK}$, pharmacokinetics; PC3, human prostate cancer cells

\section{REFERENCES}

(1) Chari, R. V.; Miller, M. L.; Widdison, W. C. Antibody-drug conjugates: an emerging concept in cancer therapy. Angew. Chem., Int. Ed. 2014, 53, 3796.

(2) Beck, A.; Reichert, J. M. Antibody-drug conjugates: present and future. MAbs 2014, 6, 15 .

(3) Agarwal, P.; Bertozzi, C. R. Site-specific antibody-drug conjugates: the nexus of bioorthogonal chemistry, protein engineering, and drug development. Bioconjugate Chem. 2015, 26, 176.

(4) Junutula, J. R.; Raab, H.; Clark, S.; Bhakta, S.; Leipold, D. D.; Weir, S.; Chen, Y.; Simpson, M.; Tsai, S. P.; Dennis, M. S.; Lu, Y.; Meng, Y. G.; Ng, C.; Yang, J.; Lee, C. C.; Duenas, E.; Gorrell, J.; Katta, V.; Kim, A.; McDorman, K.; Flagella, K.; Venook, R.; Ross, S.; Spencer, S. D.; Lee Wong, W.; Lowman, H. B.; Vandlen, R; Sliwkowski, M. X.; Scheller, R. H.; Polakis, P.; Mallet, W. Site-specific conjugation of a cytotoxic drug to an antibody improves the therapeutic index. Nat. Biotechnol. 2008, 26, 925. 
(5) Shen, B. Q.; Xu, K.; Liu, L.; Raab, H.; Bhakta, S.; Kenrick, M.; Parsons-Reponte, K. L.; Tien, J.; Yu, S. F.; Mai, E.; Li, D.; Tibbitts, J.; Baudys, J.; Saad, O. M.; Scales, S. J.; McDonald, P. J.; Hass, P. E.; Eigenbrot, C.; Nguyen, T.; Solis, W. A.; Fuji, R. N.; Flagella, K. M.; Patel, D.; Spencer, S. D.; Khawli, L. A.; Ebens, A.; Wong, W. L.; Vandlen, R.; Kaur, S.; Sliwkowski, M. X.; Scheller, R. H.; Polakis, P.; Junutula, J. R. Conjugation site modulates the in vivo stability and therapeutic activity of antibody-drug conjugates. Nat. Biotechnol. 2012, 30, 184.

(6) Thompson, P.; Fleming, R.; Bezabeh, B.; Huang, F.; Mao, S.; Chen, C.; Harper, J.; Zhong, H.; Gao, X.; Yu, X. Q.; Hinrichs, M. J.; Reed, M.; Kamal, A.; Strout, P.; Cho, S.; Woods, R.; Hollingsworth, R. E.; Dixit, R.; Wu, H.; Gao, C.; Dimasi, N. Rational design, biophysical and biological characterization of site-specific antibody-tubulysin conjugates with improved stability, efficacy and pharmacokinetics. J. Controlled Release 2016, 236, 100.

(7) Qasba, P. K. Glycans of Antibodies as a Specific Site for Drug Conjugation Using Glycosyltransferases. Bioconjugate Chem. 2015, 26, 2170.

(8) Beck, A.; Wagner-Rousset, E.; Ayoub, D.; Van Dorsselaer, A.; Sanglier-Cianferani, S. Characterization of therapeutic antibodies and related products. Anal. Chem. 2013, 85, 715.

(9) Jefferis, R. Glycosylation as a strategy to improve antibody-based therapeutics. Nat. Rev. Drug Discovery 2009, 8, 226.

(10) Jefferis, R. Glycosylation of antibody therapeutics: optimization for purpose. Methods Mol. Biol. 2009, 483, 223.

(11) Khidekel, N.; Arndt, S.; Lamarre-Vincent, N.; Lippert, A.; Poulin-Kerstien, K. G.; Ramakrishnan, B.; Qasba, P. K.; Hsieh-Wilson, L. C. A chemoenzymatic approach toward the rapid and sensitive detection of O-GlcNAc posttranslational modifications. J. Am. Chem. Soc. 2003, 125, 16162.

(12) Qu, Z.; Sharkey, R. M.; Hansen, H. J.; Shih, L. B.; Govindan, S. V.; Shen, J.; Goldenberg, D. M.; Leung, S. O. Carbohydrates engineered at antibody constant domains can be used for site-specific conjugation of drugs and chelates. J. Immunol. Methods 1998, 213, 131.

(13) Zhu, Z.; Ramakrishnan, B.; Li, J.; Wang, Y.; Feng, Y.; Prabakaran, P.; Colantonio, S.; Dyba, M. A.; Qasba, P. K.; Dimitrov, D. S. Site-specific antibody-drug conjugation through an engineered glycotransferase and a chemically reactive sugar. MAbs 2014, 6, 1190 .

(14) Zhou, Q.; Stefano, J. E.; Manning, C.; Kyazike, J.; Chen, B.; Gianolio, D. A.; Park, A.; Busch, M.; Bird, J.; Zheng, X.; SimondsMannes, H.; Kim, J.; Gregory, R. C.; Miller, R. J.; Brondyk, W. H.; Dhal, P. K.; Pan, C. Q. Site-specific antibody-drug conjugation through glycoengineering. Bioconjugate Chem. 2014, 25, 510.

(15) Li, X.; Fang, T.; Boons, G. J. Preparation of well-defined antibody-drug conjugates through glycan remodeling and strainpromoted azide-alkyne cycloadditions. Angew. Chem., Int. Ed. 2014, 53, 7179.

(16) Boeggeman, E.; Ramakrishnan, B.; Pasek, M.; Manzoni, M.; Puri, A.; Loomis, K. H.; Waybright, T. J.; Qasba, P. K. Site specific conjugation of fluoroprobes to the remodeled $\mathrm{Fc}_{\mathrm{C}} \mathrm{N}$-glycans of monoclonal antibodies using mutant glycosyltransferases: application for cell surface antigen detection. Bioconjugate Chem. 2009, 20, 1228.

(17) van Geel, R; Wijdeven, M. A.; Heesbeen, R.; Verkade, J. M.; Wasiel, A. A.; van Berkel, S. S.; van Delft, F. L. Chemoenzymatic Conjugation of Toxic Payloads to the Globally Conserved N-Glycan of Native mAbs Provides Homogeneous and Highly Efficacious Antibody-Drug Conjugates. Bioconjugate Chem. 2015, 26, 2233.

(18) Oelmann, S.; Stanley, P.; Gerardy-Schahn, R. Point mutations identified in Lec8 Chinese hamster ovary glycosylation mutants that inactivate both the UDP-galactose and CMP-sialic acid transporters. J. Biol. Chem. 2001, 276, 26291.

(19) Wright, A.; Sato, Y.; Okada, T.; Chang, K.; Endo, T.; Morrison, $\mathrm{S}$. In vivo trafficking and catabolism of IgG1 antibodies with $\mathrm{Fc}_{\mathrm{c}}$ associated carbohydrates of differing structure. Glycobiology 2000, 10, 1347.

(20) Tiberghien, A. C.; Masterson, L. A.; Patel, N. V.; Adams, L. A.; Corbett, S.; Williams, D. G.; Hartley, J. A.; Howard, P. W.; Levy, J.-N. Design and Synthesis of Tesirine, a Clinical Antibody-Drug Conjugate
Pyrrolobenzodiazepine Dimer Payload. ACS Med. Chem. Lett. 2016, DOI: 10.1021/acsmedchemlett.6b00062.

(21) Besanceney-Webler, C.; Jiang, H.; Zheng, T.; Feng, L.; Soriano del Amo, D.; Wang, W.; Klivansky, L. M.; Marlow, F. L.; Liu, Y.; Wu, $\mathrm{P}$. Increasing the efficacy of bioorthogonal click reactions for bioconjugation: a comparative study. Angew. Chem., Int. Ed. 2011, 50,8051 .

(22) Thompson, P.; Bezabeh, B.; Fleming, R.; Pruitt, M.; Mao, S.; Strout, P.; Chen, C.; Cho, S.; Zhong, H.; Wu, H.; Gao, C.; Dimasi, N. Hydrolytically Stable Site-Specific Conjugation at the N-Terminus of an Engineered Antibody. Bioconjugate Chem. 2015, 26, 2085. 